\title{
Language Iconicity from Sociolinguistic Perspective
}

\author{
Xueqing Wang \\ English department, Zhenjiang Watercraft College \\ 130 Tao Hua Wu Road, Zhenjiang 212002, Jiangsu, China \\ Tel: 86-511-8440-8158Ｅ-mail: richerwang1980@yahoo.com.cn
}

\begin{abstract}
This paper offers a definition of language iconicity. And it studies it from a fresh perspective--sociolinguistics. The paper discusses it from the following five sections: social class corresponds to language diversity; traditional concept corresponds to word order iconicity and syntactic iconicity; intimity or estrangement corresponds to lexicalization; regional varieties correspond to difference of dialect; politeness corresponds to the length of the sentence.
\end{abstract}

Keywords: Language iconicity, Sociolinguistics

\section{Introduction-Definition of Language Iconicity}

\subsection{Arbitrariness and Iconicity}

The problem how the experience of the world is converted into or mirrored in languages has impressed its marks on linguistics over centuries. The two opposite patterns appear. One holds that language and reality are quite independent of, and do not resemble each other, or in other words, language is arbitrarily structured. The other claims that language and reality must resemble each other to some extent, and language thus has the iconic nature. In essence, whether there is a natural connection between the form of a linguistic sign and what the sign depicts has been the focus of the dispute. Ferdinand de Saussure asserted that the link between the signifier and the signified is arbitrary. That is to say, the form of most words (or linguistic signs) is linked to what they signify only by convention; the sign is arbitrary in the sense that there is no inherent association between sound and meaning. The paradigm initiated by Saussure has prevailed historically. The principle of arbitrariness is widely believed to begin with Saussure in 1916, continue with Bloomfield and cultivate with Chomsky. In Cours de Linguistigue Ginerale, arbitrariness was regarded as the first principle of language. For Saussure, a sign is a unity of sound image and concept inseparable like the two sides of a coin. According to him, there is no intrinsic or logical connection between them. In other words, they relate to each other arbitrarily.

The extreme witness is found in Chomsky's Language and Mind (1968). For him, structural arbitrariness is the hallmark of human language in contrast with the more iconic animal communication.

...Every animal communication system that is known...either consists of a fixed, finite number of signals, each associated with a specific range of behavior...or it makes use of a fixed, finite number of linguistic dimensions, each of which is associated with a particular non-linguistic dimension in such a way that selection of a point along the linguistic dimension determine and signals a point along the associated non-linguistic dimension. The mechanism and principle, however, are entirely different from those employed by human language... (Chomsky, 1968: 69)

In the following years, Chomsky's been undoubtedly consistent with the nature of language. Some credo has been found in his scattered writing as follows:

Our interpretation of the world is based in part on representational systems that derive from the structure of the mind itself and do not mirror in any direct way the form of things in the external world (Chomsky, 1981: 3).

\subsection{Peirce's view of iconicity}

During the last two decades, however, this rigid view of the linguistic sign has come under increasing criticism. Contrary to the Saussurean idea that language is fundamentally if not exclusively arbitrary (or in semiotic terms, 'symbolic'), considerable linguistic research in the twentieth century has shown that iconicity operates in practically every known language. In this context the idea of C. S. Peirce, a nineteenth-century American philosopher, has been revived. Peirce stated "...in the syntax of every language there are logical icons of the kind that are aided by conventional rules..." In this sense, Peirce challenged the traditional idea that iconicity is only shown in onomatopoeia by classifying iconicity into two types. In his taxonomy of icon, Peirce made a crucial 
distinction between two types of iconicity, i.e. image iconicity and diagram iconicity. An icon image is a single sign that resembles its referent with respect to characteristics. An iconic diagram is a systematic arrangement of signs; none of which necessarily resembles its referent, but whose relationships to each other mirror the relationships of their referents. The focal point of the two types of icons is the similarity between a sign and what it stands for, which lies in either the matching parts in an iconic image or the relationship among the parts in an iconic diagram.

In a word, iconicity as a semiotic notion refers to a natural resemblance or analogy between the form of a sign ('the signifier'--a letter or sound, a word, a structure of words, or even the absence of a sign) and the object or concept ('the signified') it refers to in the world or rather in our perception of the world.

\section{Sociolinguistic Perspective}

As we all know, sociolinguistics was initiated by an American linguist H.Currie in 1950s. In the following years, it has become an independent language science. In sociolinguists' view, language belongs to the whole system of society. All kinds of social situations, relationships and social phenomena can be mirrored in language. Actually, there is an iconicity model called "society--language".

Sociolinguistics is a term including the aspects of linguistics applied toward the connections between language and society, and the way we use it in different social situations. Sociolinguists assert that it is the study of the relationship between language and the social forms, structures, functions, and processes in which it is used. It explores issues about the form and use of language that pertain to the smaller unite of social organization (e.g., individuals, gender groups, and neighborhoods) and more micro-levels of the language in use (e.g., phonemes, address forms, conversational units etc).

\subsection{Social class corresponds to language diversity}

Studies indicate that language diversity (including pronunciation, words, syntax and so on) enlarges along with the enlargement of the social class. Inevitably whether high or low the social rank is will lay traces on the literal communication, including speaking and writing. It is said that people from higher social class always choose more refined and standard words than guys of lower rank do. Sociolinguist Trugill said (1982; 34) that the internal differentiation of human societies is reflected in their languages. After the study of the different people's choices of using language, Bernstein, a British sociologist, differentiates two kinds of codes: Elaborated Code and Restricted code. And he also asserts that the syntactical structure of the former code is more complicated than that of the latter; adjectives could be more easily seen in the former than in the latter. It is reported that children from middle class families prefer to use these two codes while those from labor class would rather choose the latter one. A more interesting research made by Labov (1966,p.240) is that in New York City English, The $r$-lessness is most characteristic of the lower and working classes. A man can be easily classified into whether higher class or lower rank according to the usage of the $r$-lessness in his English.

A typical example quoted by a famous Chinese linguist Wang Dechun is that when people of higher rank and with higher education receive foreign words, they always take them according to their transliteration. While the rural people or people with lower education background would never do. An instance is that many Chinese in urban areas would unconsciously say "bye, bye, OK" when communicating with others, whereas people from outskirt seldom use.

In addition, whether a person would always like to use colloquialism or not can reflect his social class together with his education background. There is a good saying in Chinese "san ju hua bu li ben hang", which is typical of it.

There are more examples about the iconicity between social class and language diversity: First, in ancient feudal China, some words are privileged to king families. Any ordinary grass-tooted people offend to use them; he would take a risk of being slaughtered. Second, in another Asian country-India, there is a strict caste. Different people from different castes use gorgeously different words. Third, people, when speaking, usually have a tendency that they choose formal, mannerly language toward others with higher classes.

\subsection{Traditional concept corresponds to word order iconicity and syntactic iconicity}

It is not uncommon for the order of morphemes, words or sentences to reflect logical relations among their referents. In dealing with the principle of linear order, Givon(1994) claims that the order of clauses in a coherent discourse will tend to correspond to the temporal order of the occurrence of the depicted events. An obvious exemplification is the clauses ordered according to the temporal sequence of the events. The narrative sequence of "veni, vidi, vici" (I came, I saw, I conquered), a well-known saying by Caesar has been adduced by Jakobson of a typical iconic structure, for the sequence of the three elements tallies with the order of historical events. 
From the perspective of word order, social relationship in traditional Chinese culture is a certain one with nobleness over lowliness for example: in Chinese ,we say "shang xia;da xiao gao di;tian di;qian hou;xian hou;lao shao;zhang you;zun bei;zuo you". More examples are "da da xiao xiao;qian qian hou hou;yi qian yi hou;gao bu cheng,di bu jiu;zuo gu you pan; tian shi,di li,ren he;da zhong xing qi ye;zhong xiao xue ......". In language gender, the traditional thought that male is superior to female also lays its trace on language. We say in Chinese that "nan nan $n v$ nv;nan nv lao shao;nan da dang hun,nv da dang jia;nan nv ping deng;fu mu;xiong mei;ge ge jie jie......".In western culture ,although there is a custom called "lady first", in language of English "man and woman, boys and girls, Adam and Eve" and so on can also be heard.

From the perspective of syntax, syntactic order of Chinese looks like a triangle upside down $(\nabla)$ :the more important and more complicated one is put ahead.(Wang Yin 1990,1992) And syntactic order of English is like a triangle $(\triangle)$ :the less important and less complicated one is set ahead. The end of an English sentence seems to be the most important place of sending a message.

Examples:

\section{(1) jiu si fu shang}

Cure the wounded and save the dying

There is no doubt that in Chinese the dying is emergent than the wounded. So "si" is put ahead of "shang".

\section{(2) No 40 Broadway, New York, United States of America}

Mei guo, niu yue, bai lao huida jie 402 hao

(3) He was born in a small village near Jinan, Shandong Province, People's Republic of China.

Ta chu sheng zai zhong guoshan dong sheng ji nan fu jin de yi ge xiao cun zhuang.

\subsection{Intimity or estrangement corresponds to lexicalization}

In 1982, Trudgill said that the intimate relatives have got proper nouns to describe, while distant ones have not. Son, daughter, father, mother, etc express the intimity. Son-in-law, daughter-in-law, the wife of my husband's brother(zhou li).In Chinese culture, it is possible for the wives of one's husband's brothers to live together. Definitely they are intimate. So they get a special lexical word of their own. However, in western culture, a new couple would like to move out to live independently. So it is not necessary to create a proper noun for that relationship. "qin jia gong, qin jia mu" can also be heard in Chinese communities because in Chinese background this kind of personal relationship seems intimate, whereas in the language of English, the corresponding noun can never hardly be found. "The fathers of husband and wife, the mothers of husband and wife" should possibly become the most proper noun for describing this relationship in western culture. An instance from Russian is that the word "shurin" equaled the meaning of wife's brother in the middle of $19^{\text {th }}$ century, because that century, wife's brother lived with the whole family as a union. Later on, when they did not live together, they became more and more estranged. So that lexical word of "shurin" could never find any reason to exist. A new phrase "brat zheny" came into being. A lexical word disappears along with the disappearance of the significance of the relationship.

An interesting language phenomenon is that there are only two words in English----"brother" and "sister" without differentiating who is elder or younger. However, in Chinese ,we've got "ge ge,jie jie, di di, mei mei", at least four different words. This difference surely indicates that the need of social communication also corresponds to lexicalization. In China, elder brother and elder sister should inevitably shoulder more responsibility of taking care of the family and the younger. Another example is that the word "cousin" in English equals to altogether 12 corresponding relatives, who are "tang xiong di jie mei, yi xiong di jie mei, biao xiong di jie mei" in China. It's not necessary for English culture to distinguish those cousins whether come from fathers' side or mothers' side, though that kind of division seems very important in Chinese culture. All the titles such as "da jie, er di, san sao, wu ma, liu ye, qi gu, ba yi" can be looked as the epitomes of iconicity between the social communication and language.

\subsection{Regional varieties correspond to difference of dialect}

The study of dialect has been the hot topic of sociolinguistics. Linguists also feel that iconicity can be touched between dialect and regional distance. That is to say there is a direct ratio between these two--the farther the regional distance is, the bigger the difference of dialect will be. Take two instances: English that Buchan from north Scotland use differs largely from that of London English. In China, people from North can communicate fluently in their own dialect with those from Shandong Province in theirs. However, there will be trouble when a person from North and the other from Guangdong Province meet and talk to each other with their respective 
local dialects.

From the region, one's dialect can be deduced; on the other hand, from the dialect, one can also be reasoned which region he belong to. From the sociolinguistics, a sentence that "A man is known by the dialect he speaks" get proved.

\subsection{Politeness corresponds to the length of the sentence}

In many languages, sentences expressing politeness are longer than those not. And the sentence structure of the former is much more complicated than that of the latter. In Chinese, the word expressing the singular form of the second person include“你” and “您”. The latter is far complex than the former not only in usage but in structure.

The following sentences all express the same meaning - open the door. However, the tone differs much from one to another. Generally speaking, the more words a sentence has, the more complicated the structure of the sentence is and the more polite the tone will be.

(4) Open the door.

(5) Please open the door.

(6) Will you open the door?

(7) Would you please open the door?

(8) Would you mind if I open the door?

(9) Would you please be kind enough to open the door?

From this perspective, it is not difficult to understand why subjunctive mood is more polite than ordinary tone in English.

Chinese seems contrary to English. Language used by upper class with higher education background is affected by old Chinese. Such words as "zhi hu zhe ye" are usually picked by upper classes. As time goes by, idioms, phrases and parallel structures are frequently used.

\section{Conclusion}

Iconicity has contributed much to the study of language, concept and society. The research should not be clinged on to onomatopoeia, neither should it be stuck to cognitive linguistics. Sociolinguistics offers a fresh angle to look at it. The research in this paper is just a beginning and it is far from ending.

\section{References}

Haiman, John. (1985). Natural Syntax: Iconicity and Erosion. Cambridge University Press.

Hawkins, J. (1983). Word Order Universals. New York: Academic Press.

Newmeyer, F. J. (1992). Iconicity and generative grammar. Language, (68):756-796.

R. A. Hudson. (2000). Sociolinguistics. Foreign Language Teaching and research Press \& Cambridge University Press.

Stern, H. H. (1997). Fundamental concepts of Language Teaching. Shanghai Foreign Language And Education Press.

Trudgill. (1982). Sociolinguistics. New York: Penguin Books.

Ungerer, F \& Schmid, H. J. (2001). An Introduction to Cognitive Linguistics. Foreign Language Teaching and research Press. 